\title{
Normal Tritan Discrimination in Amblyopia Suggests Preservation of Koniocellular Function
}

\author{
Ross Littlewood \\ Midland Eye Clinic, Midland, Australia \\ Email address: \\ idoc@westnet.com.au \\ To cite this article: \\ Ross Littlewood. Normal Tritan Discrimination in Amblyopia Suggests Preservation of Koniocellular Function. International Journal of \\ Ophthalmology \& Visual Science. Vol. 3, No. 3, 2018, pp. 43-46. doi: 10.11648/j.ijovs.20180303.12
}

Received: October 9, 2018; Accepted: October 23, 2018; Published: November 15, 2018

\begin{abstract}
Background: Human stereoscopic vision is a psychological abstraction emerging from sufficiently grouped monocular signals within a broader unity of simultaneous binocular perception. Incongruent central signals inevitably arise when moving through a visual landscape, due to changing asymmetries of perception, producing signal rivalry. Humans have an evolved capacity to dynamically inhibit incongruous or ambiguous signals in order to maintain a coherent unity of stereoscopic perception, a facility sometimes exploited by optical illusions. Unlike the beneficial mental construct that arises from physiological inhibition, the amblyopic inhibition is permanently unilaterally destructive, has a large field size, is associated with irreversible signal inhibition at the dorsal lateral geniculate nucleus (dLGN), and leads to structural alterations in dLGN that can be demonstrated histologically. Recent reports suggest amblyopic signal inhibition at the dLGN may be selective, preferentially involving the parvocellular system and sparing the koniocellular system. It is not yet known whether physiological inhibition is also pathway specific, but it would be surprising if it were not. The two chromatic opponent systems display differing vulnerabilities in a number of disease states, including optic neuritis and toxic neuropathies, and they followed independent evolutionary paths during phylogenetic development from small primates into apes and humans with larger eyes. Tritan contrast is conducted exclusively by the koniocellular system as far as the striate cortex, so clinical tests of tritan contrast may provide information about koniocellular function in humans. If koniocellular function is not inhibited in amblyopia then tritan discrimination tests may be normal in amblyopic eyes. Objective: In this study the $\mathrm{C}$ test for tritan discrimination was employed as a proxy for koniocellular function to discover whether tritan discrimination was affected in proportion with achromatic contrast in the amblyopic eye of 18 amblyopic adults. Method: The tritan contrast and logmar visual acuity results were compared between eyes, using two non parametric statistical tests. Results: The $\mathrm{C}$ test scores for tritan discrimination were statistically similar in the normal and amblyopic eyes, but mean logmar acuity scores were significantly different, with a mean of 0.76 in the amblyopic eyes compared to 0.18 in the non-amblyopic eyes. Conclusion: This finding is consistent with preservation of koniocellular signal perception despite parvocellular signal inhibition in amblyopic eyes.
\end{abstract}

Keywords: Amblyopia, Koniocellular, Tritan, C Test

\section{Introduction}

The koniocellular ganglion cell pathway is functionally and anatomically discrete from the parvocellular pathway in the lateral geniculate nucleus and striate visual cortex of primates [1]. There is significant cortico-geniculate inhibition that modulates parvocellular activity at the dorsal lateral geniculate nucleus (dLGN) [2] but the structural components and possible selectivity of this inhibition are not understood [3]. Functional magnetic resonance imaging (fMRI) of amblyopic patients has shown decreased activation in the lateral geniculate nucleus (LGN) when the amblyopic eye is used $[4,5]$. The koniocellular fibres of the geniculo-cortical pathway terminate in different layers of V1 than the parvocellular fibres, as well as directly in extra-striate cortex, and there are also koniocellular projections to the middle temporal area V5 $[1,6]$, potentially contributing to the non-conscious visual discrimination observed in some cases of cortical blindness (blind vision).

Analysis of chromatic opponent responses of the LGN in amblyopic adults shows selectively more decrease in activation when the opponent chromatic signal selects for 
parvocellular rather than koniocellular activation [7]. A clinical test responding to tritan contrast provides a proxy of koniocellular function. If koniocellular function is inhibited in an amblyopic eye then tritan discrimination should be reduced, and vice versa.

The $\mathrm{C}$ test for tritan discrimination is a novel plate test that provides an ordinal score from 1 to 10 , corresponding to the perception of sequentially decreasing amounts of tritan contrast [8]. Because tritan contrast decreases with increasing grades of nuclear sclerosis, plate tests for tritan contrast require stronger daylight illumination than red/green plate tests in older observers. I have found it necessary to employ 800 lux of artificial daylight to ensure the full dynamic range of the $\mathrm{C}$ test is available in older phakic subjects, and also to perform a LOCS III [9] grading of nuclear colour (NC) [8]. Reliable normative test data has only been published for grades of $\mathrm{NC}<4$, and in those cases the expected $\mathrm{C}$ test score in a normal eye is $\geq 8$.

\section{Methods}

During a 4 month period all patients presenting to an adult ophthalmology clinic had their routine clinical findings (including logmar acuity and a $\mathrm{C}$ test score) recorded in an Excel spreadsheet as part of a study to establish normative results for the $\mathrm{C}$ test. The results obtained from the first 243 observers were published [8] then a further 149 subjects were assessed in an attempt to establish normal scores for eyes with $\mathrm{NC}>4$. During the test evaluation process I observed that amblyopic eyes had high $\mathrm{C}$ test scores, despite poor logmar acuity, and this incidental observation prompted the current analysis. A retrospective analysis of the entire data set found data from 18 amblyopic subjects with $\mathrm{NC}<4$ in both eyes, and an inter-ocular comparison of that data is reported here. The data was ordinal but non-parametric, with a small sample size, so non parametric statistical analysis was performed using the Mann-Whitney U test and Kruskal-Wallis test.

\section{Results}

The age range was 46 to 78 years and 12 subjects were males. There were two pseudophakic eyes in both groups, indicated by zero values in their NC grading. Amongst the amblyopic eyes the mean logmar acuity was 0.76 and the median 0.74 , and among the non-amblyopic eyes the mean and median were both 0.18 . A comparison of the logmar acuities between the two sets of acuity results showed a statistically significant difference which is evident in table 1 . A Mann-Whitney $U$ test comparing the two distributions confirmed that the difference is statistically significant $(\mathrm{Z}$ score $=5.1096, \mathrm{p}<0.00001)$.

A comparison of the $\mathrm{C}$ test results between the amblyopic and non-amblyopic eyes showed no significant difference. The amblyopic eyes had a mean $\mathrm{C}$ score of 9.3 and a median of 9.0. The non-amblyopic eyes had a mean $\mathrm{C}$ score of 9.4 and a median of 9.5. A Mann-Whitney U test comparing the two sets of C score data showed no significant difference $(\mathrm{Z}$-score $=-0.3797, \mathrm{p}=$ 0.704). A Kruskal-Wallis analysis was also performed on the $C$ score results and showed no significant difference between the two groups (H statistic $=0.1564, \mathrm{p}=0.6925)$.

Table 1. Data from 18 adults with one amblyopic and one normal eye, showing cause of amblyopia, best corrected Logmar acuity, LOCS III nuclear colour (1 6), and $C$ test score $(1-10)$.

\begin{tabular}{|c|c|c|c|c|c|c|c|c|}
\hline & \multirow{2}{*}{ DIAGNOSIS } & \multirow{2}{*}{ AGE } & \multicolumn{3}{|c|}{ AMBLYOPIC EYE } & \multicolumn{3}{|c|}{ NON - AMBLYOPIC EYE } \\
\hline & & & Logmar acuity & Nuclear color & C score & Logmar acuity & Nuclear color & C score \\
\hline 1 & R exotropia & 78 & 0.88 & 0 & 9 & 0.20 & 3 & 9 \\
\hline 2 & R esotropia & 63 & 0.54 & 2 & 9 & 0.26 & 2 & 10 \\
\hline 3 & L esotropia & 53 & 0.86 & 1 & 9 & 0.32 & 1 & 9 \\
\hline 4 & $\mathrm{~L}$ anisometropia & 69 & 0.72 & 2 & 10 & 0.18 & 2 & 10 \\
\hline 5 & R esotropia & 71 & 0.68 & 2 & 9 & 0.22 & 3 & 8 \\
\hline 6 & L esotropia & 75 & 0.64 & 3 & 9 & 0.18 & 2 & 10 \\
\hline 7 & L esotropia & 64 & 0.92 & 3 & 9 & 0.32 & 3 & 9 \\
\hline 8 & L hypertropia & 53 & 0.76 & 2 & 10 & 0.16 & 1 & 10 \\
\hline 9 & $\mathrm{R}$ esotropia & 76 & 0.74 & 0 & 10 & 0.18 & 0 & 10 \\
\hline 10 & L esotropia & 73 & 0.66 & 2 & 9 & 0.22 & 2 & 9 \\
\hline 11 & L exotropia & 59 & 0.66 & 2 & 9 & 0.12 & 3 & 10 \\
\hline 12 & $\mathrm{R}$ anisometropia & 60 & 0.76 & 2 & 10 & 0.12 & 2 & 9 \\
\hline 13 & R exotropia & 74 & 0.72 & 3 & 9 & 0.28 & 3 & 9 \\
\hline 14 & R esotropia & 50 & 0.92 & 1 & 10 & 0.08 & 2 & 9 \\
\hline 15 & L esotropia & 72 & 0.74 & 3 & 9 & 0.08 & 0 & 10 \\
\hline 16 & L hypertropia & 62 & 0.88 & 2 & 9 & 0.12 & 3 & 10 \\
\hline 17 & R exotropia & 46 & 0.88 & 1 & 10 & 0.06 & 1 & 10 \\
\hline \multirow[t]{2}{*}{18} & L esotropia & 74 & 0.64 & 3 & 9 & 0.22 & 3 & 9 \\
\hline & MEAN & 65 & 0.77 & 1.9 & 9.3 & 0.18 & 2.0 & 9.4 \\
\hline
\end{tabular}

\section{Discussion}

Each $\mathrm{C}$ test plate consists of a foreground circle interrupted by a gap, superficially resembling a Landolt $\mathrm{C}$ rotated into one of 8 orientations (figure 1). The symbol differs importantly from a Landolt $\mathrm{C}$ because vertical and horizontal contrast has been removed in order to minimise the risk of unwanted parvocellular perception. Observers usually see the location of the break in the $\mathrm{C}$ at a glance when the contrast is high, but at low contrast the mode of perception changes. If an observer fails to immediately perceive the target they are instructed to 
move their gaze around the foreground circle and attempt to discover the break dynamically. Using this strategy most observes pass an extra one or two plates they previously failed. Because the foreground is isoluminant the movement is unlikely to produce a magnocellular response [10]. The results of the present study show that tritan contrast measured by the $\mathrm{C}$ test is better preserved than achromatic contrast measured by logmar acuity. This is consistent with retention of the koniocellular signal in the presence of selective suppression of the parvocellular signal in amblyopic eyes.

One of the cardinal features distinguishing primates from other vertebrates is forward directed orbits and binocular vision enabling stereoscopic depth perception. Comparative data from different primate species suggests that high level stereoscopic vision emerges exclusively from parvocellular signals that have the necessary spatial resolution [11] to provide both fine and coarse interocular differences in perspective [12]. Parvocellular signalling in humans is an L/M opponent process, since the central fovea is relatively $\mathrm{S}$ cone free in large primates and humans. The dependence on $\mathrm{L} / \mathrm{M}$ contrast is an unavoidable adaptation to scatter and dispersion of short wavelengths within long eyes [13]. The evolution of stereopsis required an excitatory signal in response to sufficient grouping of high resolution signals from the central field of vision, but also an inhibitory signal to resolve non-congruous signal components otherwise perceived as retinal rivalry [14]. The central field of vision with its midget ganglion cell population provides a high resolution parvocellular signal in humans, whilst the koniocellular response is predominantly paracentral with larger receptive fields.

Amblyopia in the context of ocular misalignment arises under conditions where excitatory signals from grouping are not available, and inhibition is useful for resolving retinal rivalry. Amblyopia in the context of visual deprivation or refractive error also arises under conditions where the parvocellular inputs are dissimilar. Neither the magnocellular or koniocellular pathways have sufficient spatial resolution to mediate high level stereopsis, so inhibition of the parvocellular signal alone may be sufficient to eliminate signal rivalry or ambiguity, and enable coherent perception.

Subjects with occipital lobe blindness may have Riddoch's syndrome in which fMRI scans reveal persisting visual discrimination without visual consciousness [15]. Such subjects retain extra striate visual input, which is not parvocellular. Movement detection, directed visual attention, and a recordable visual evoked response have been found in these subjects [16]. Some of these observations are best explained by retention of koniocellular or magnocellular signalling [10]. In contrast to Riddoch's syndrome, amblyopia involves an incomplete central absence of the parvocellular signal, but visual function in amblyopia shares some characteristics described in Riddoch's syndrome, such as low spatial resolutions inhibited by background noise, a characteristic of the koniocellular pathway [17].
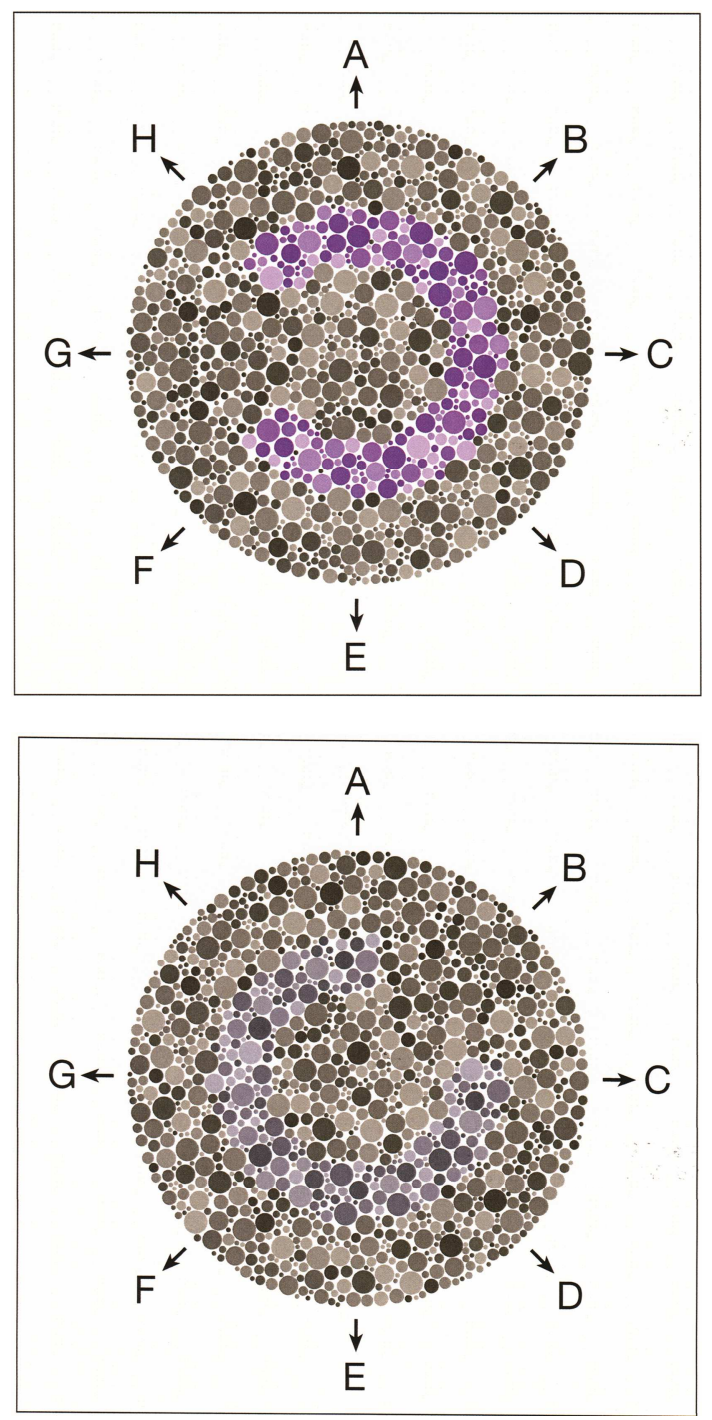

Figure 1. C test plates 1 (top) and 8 (bottom).

\section{Conclusion}

The normal $\mathrm{C}$ test response in amblyopic eyes is a useful confirmation that the $\mathrm{C}$ test design isolates the koniocellular from the parvocellular response. Most readily available clinical tests of tritan function require the subject to either consciously categorise a color, or identify a geometrical pattern, both of which require a contingent parvocellular response in order to achieve a detailed conscious perception. Further testing would be required to determine which specific tritan tests are similarly preserved in amblyopia. The $\mathrm{C}$ test may be useful in clinical situations where the explanation of visual loss is ambiguous, provided that $\mathrm{NC}<4$. Wider use of tritan testing in clinical practice would provide a more complete understanding of visual perception and counteract the current trend in ophthalmology towards 'parvocellular perfectionism'. Tritan testing has specific application in a range of optic neuropathies, as an aid to providing a prognosis in ischemic retinal diseases, in unexplained vision loss when there is no history of amblyopia, and as an ancillary test in the evaluation of cataract. 


\section{Conflicts of Interest}

The author declares there are no competing interests.

\section{References}

[1] S. H. Hendry, and R. Clay Reid, (2000). "The Koniocellular pathway in primate vision," Ann Rev Neurosci, Vol. 23, 2000, pp. 127-153.

[2] M. H. Mobarhan, G. Halnes, P. Martinez-Cañada, T. Hafting, M. Fyhn, and G. T. Einovoll, "Firing-rate based network modelling of the dLGN circuit: Effects of cortical feedback on spatiotemporal response properties of relay cells," Plos Comput Biol, Vol. 14, No. 5, 2018, e1006156.

[3] J. J. Nassi, and E. M. Callaway, "Parallel processing strategies of the primate visual system," Nat Rev Neurosci, Vol. 10, 2009, pp. 360-372.

[4] R. F. Hess, B. Thompson, G. A. Gole, and K. T. Mullen, "Deficient responses from the lateral geniculate nucleus in humans with amblyopia," Eur J Neurosci, Vol. 29, No. 5, 2009, pp. 1064-70.

[5] A. Miki, G. T. Liu, Z. G. Goldsmith, C. S. Liu, and J. C. Haselgrove, "Decreased activation of the lateral geniculate nucleus in a patient with anisometropic amblyopia demonstrated by functional magnetic resonance imaging," Ophthalmologica, Vol. 217, No. 5, 2003, pp. 365-9.

[6] L. C. Sincich, K. F. Park, M. J. Wohlgemuth, and J. C. Horton "Bypassing V1: a direct geniculate input to area MT," Nat Neurosci, Vol. 7, 2004, pp. 1123-1128.

[7] R. F. Hess, B. Thompson, G. A. Gole, and K. T. Mullen, "The amblyopic deficit and its relationship to geniculo-cortical processing streams," J Neurophysiol, Vol. 104, No. 1, 2010, pp. 475-83.
[8] R. Littlewood, and F. Hyde, "The "C test" for tritan discrimination," Color Res Appl, Vol. 43, No. 1, 2018, pp. 58-64.

[9] L. T. Chylak, J. K. Wolfe, D. M. Singer, M. C. Leske, M. A. Bullimore, I. L. Bailey, J. Friend, D. McCarthy, and S. Y. Wu, "The Lens Opacities Classification System III," JAMA Ophthalmology, Vol. 111, No. 6, 1993, pp. 831-836.

[10] A. Cheng, U. T. Eysel, and T. R. Vidyasagar, "The role of the magnocellular pathway in serial deployment of visual attention," Eur J Neurosci, Vol. 20, No. 8, 2004, pp. 2188-2192.

[11] R. A. Barton, "Binocularity and brain evolution in primates," $P$ Natl Acad Sci USA, Vol. 101, No. 27, 2004, pp. 10113-10115.

[12] D. Giaschi, S. Narasimhan, A. Solski, E. Harrison, and L. M. Wilcox, "On the typical development of stereopsis: Fine and coarse processing," Vision Research, Vol. 89, 2013, pp. 65-71.

[13] C. A. Curcio, K. A. Allen, K. R. Sloan, C. L. Lerea, J. B. Hurley, I. B. Klock, and A. H. Milam, "Distribution and morphology of human cone photoreceptors stained with anti-blue opsin," $J$ Comp Neurol, Vol. 312, No. 4, 1991, pp. 610-624.

[14] F. Tong, M. Meng, and R. Blake, "Neural bases of binocular rivalry," Trends Cogn Sci, Vol. 10, No. 11, 2006, pp. 502-511.

[15] S. Zeki and D. H. Ffytche, "The Riddoch syndrome: insights into the neurobiology of conscious vision," Brain, Vol. 121, No. 1, 1998, pp. 25-45.

[16] J. Sanchez-Lopez, C. A. Pedersini, F. Di Russo, N. Cardobi, C. Fonte, V. Varalta, M. Prior, N. Smania, S. Savazzi, and C. A. Marzi, "Visually evoked responses from the blind field of hemianopic patients," Neuropsychologia, Vol. 17, 2017, pp. 30377-9.

[17] D. M. Levi, C. Yu, S. G. Kuai, and E. Rislove, "Global contour processing in amblyopia," Vision Research, Vol. 47, No. 4, 2007, pp. 512-524. 